\title{
Cocobolo-Holzallergie bei einer Flötenlehrerin
}

\author{
Contact Allergy to a Cocobolo Recorder
}

\section{Zusammenfassung}

Eine 62-jährige Flötenlehrerin entwickelte ein Jahr nach der Verwendung einer neuen dekorativen Blockflöte eine Schwellung der Unterlippe, Konjunktivitis, Kribbeln und Juckreiz bis in den Hals hinein, Atembeschwerden und das Gefühl immer husten zu müssen. Ekzematische Veränderungen zeigten sich auch am Kinn. Im Epikutantest reagierte sie in der 72-Stundenablesung schwach auf (R)-4-Methoxydalbergion und stark auf Obtusachinon, die typischen Inhaltsstoffe des Cocoboloholzes Dalbergia spp. „Mittelamerika“. In den darauf folgenden Tagen verstärkten sich die Testreaktionen, die Lippe schwoll erneut an. Der Flötenhersteller bestätigte die verwendete Holzart als Cocobolo. Ein kurzer Aufenthalt in dessen Werkstatt zwecks Anfertigung eines Ersatzmundstückes rief bei der Patientin am folgenden Tag ein Rezidiv hervor. Seit Meiden des Instruments ist die Lehrerin erscheinungsfrei. Vier weitere, bisher unveröffentlichte Fälle einer Kontaktallergie durch Cocoboloholz werden kurz vorgestellt (Geigenkinnhalter, Holzarmband, Pistolengriff, Einbaumöbel). Obwohl Cocobolo nur für wenige Zwecke eingesetzt wird, zeigen der beschriebene und die erwähnten Fälle erneut, wie ernst seine sensibilisierenden Inhaltsstoffe zu nehmen sind.

\section{Abstract}

A 62-year-old female recorder teacher developed swelling of her lower-lip, conjunctivitis, itching of the throat, enforced coughing and eczematous reactions at the lower jaw. The recorder was made from Cocobolo wood (Dalbergia spp. „Central America“). Epicutaneous tests produced a weak reaction to (R)-4-methoxy dalbergion and a strong one to obtusaquinone, both known constituents of this species. Due to the tests the lower-lip again became swollen on the following days. A request for replacement of the mouthpiece with a different wood was refused by the recorder manufacturer. Instead he painted the outer part of the mouthpiece with a durable lacquer to keep the good sound of the instrument. However the patient did not dare to play this recorder again but changed to other ones out of the 24 instruments she owned. No relapse has been encountered ever since. To throw a light on the high sensitizing properties of this Dalbergia species four further cases are shortly described that remained unpublished up to date. One occurred in a cabinet maker, the others were due to contact with a violin chin rest, a wooden bracelet and the pistol grip of an artistic sportsman.

\section{Einleitung}

Trotz der untergeordneten Rolle, die der Musikunterricht heute noch in unseren Schulen spielt, gibt es doch kaum ein Kind, das nicht wenigstens einmal in seinem Leben ein paar Stunden Blockflötenunterricht genommen hat. Dementsprechend gibt es auch eine kleine Zahl von Lehrern und Lehrerinnen, die Unterricht erteilen. Blockflöten aus Kunststoff oder einer einheimi- 
schen Holzart sind günstig zu erstehen. Sie werden jedoch achtlos liegen gelassen, wenn die Freude am Blockflötenspiel erlischt oder das Kind in eine höhere Klasse kommt und sich anderen Interessen zuwendet. Anders ist es mit denjenigen, die ihren Lebensunterhalt mit dem Unterricht verdienen und daher auf ein gutes, d.h. teureres Instrument zurückgreifen. Unterrichtende besitzen häufig mehrere Blockflöten, doch unter diesen befindet sich eine, die sie besonders gerne spielen: die Cocoboloflöte. Diese Zuwendung hat ihren besonderen Grund. Die Blockflöte aus Cocobolo sieht nicht nur sehr dekorativ aus, besitzt hervorragende physikalische und besondere Klangeigenschaften, sondern muss auch vor dem Unterricht kaum eingespielt werden. Bei dieser Flöte ist es nicht notwendig, eine Viertelstunde „Vorlauf“ zu geben, um sie dann auf die richtige Tonhöhe einstellen zu können. Der Kern des Cocoboloholzes (Dalbergia spc. „Mittelamerika“, Familie Fabaceae) ist prall mit Inhaltsstoffen gefüllt, gelblich über orange bis violettbraun gefärbt, oft mit einer violetten bis schwarzen Streifung versehen und später tief rotbraun nachdunkelnd. Es zeigt in seinen Poren dunkle, glänzende Einlagerungen, die das Gefäß in seiner ganzen Länge verstopfen. Die dadurch bedingte äußerst geringe Wasseraufnahme, zusammen mit seiner dichten Oberfläche, hohen Härte und guten Bearbeitbarkeit machen es zu einem der besten Flötenhölzer der Welt [1]. Dank seiner aromatischen Inhaltsstoffe (Dalbergione und verwandte Verbindungen) ist das Holz so pilz- und insektenresistent, dass es sich auch hervorragend als Wasserbauholz eignet [2]. Trotz der seit über hundert Jahren bekannten, krank machenden Eigenschaften dieses Holzes werden bis heute allergische Kontaktdermatitiden beobachtet, weil Cocobolo durch kein gleichwertiges anderes Holz ersetzt werden kann. Man verwendet es wegen der genannten Eigenschaften nach wie vor für Blockflöten und Teile anderer, hochwertiger Blasinstrumente (z. B. Klarinettenbecher), mechanisch beanspruchte Teile von Streich- und Zupfinstrumenten (z.B. Gitarrenstege) sowie für Billardstöcke, Holzschmuck (Armbänder, Holzketten u. a. Gegenstände des Kunstgewerbes), Griffe und Griffschalen, Intarsien, Bürstenrücken und gelegentlich im Innenausbau. In dem hier vorgestellten Fall handelte es sich um eine Flötenlehrerin.

\section{Kausistik}

Im August 2002 stellte sich eine 62-jährige Lehrerin, die seit 27 Jahren den Beruf einer Flöten- und Gitarrenlehrerin ausübt, mit Schwellungen und feinlamellärer Schuppung an den Lippen vor, die nicht abheilten. Sie berichtete über Juckreiz und Kribbeln an den Augen, der Nase und der Zunge bis in den Hals hinein, Atembeschwerden und das Gefühl, ständig abhusten zu müssen. An der rechten Stirnseite zeigte sich eine $5 \mathrm{~mm}$ große, scharf begrenzte Macula. An der linken Wange fand sich eine $5 \mathrm{~mm}$ große, homogen hellbraun pigmentierte Lentigo senilis mit einem ca. $1 \mathrm{~mm}$ großen dunkleren „Einsprengsel“. Gegenüber der Oberlippe war die Unterlippe ungefähr um das Vierfache angeschwollen (Abb.1). Von dort ausgehend setzten sich die ekzematischen Veränderungen bis zum Unterrand des Kinns fort (Abb. 2).

Die Patientin brachte zur Untersuchung eine Tasche voller Instrumente mit. Aus diesen 26 Palisander-, Rosen-, Kirschen-, Eben- und Ahornholzflöten fischte sie eine sehr dekorative heraus, die sie am ehesten als Ursache ihrer Hautveränderungen



Abb. 1 Schwellung der Unterlippe nach Spielen der verdächtigten Blockflöte.

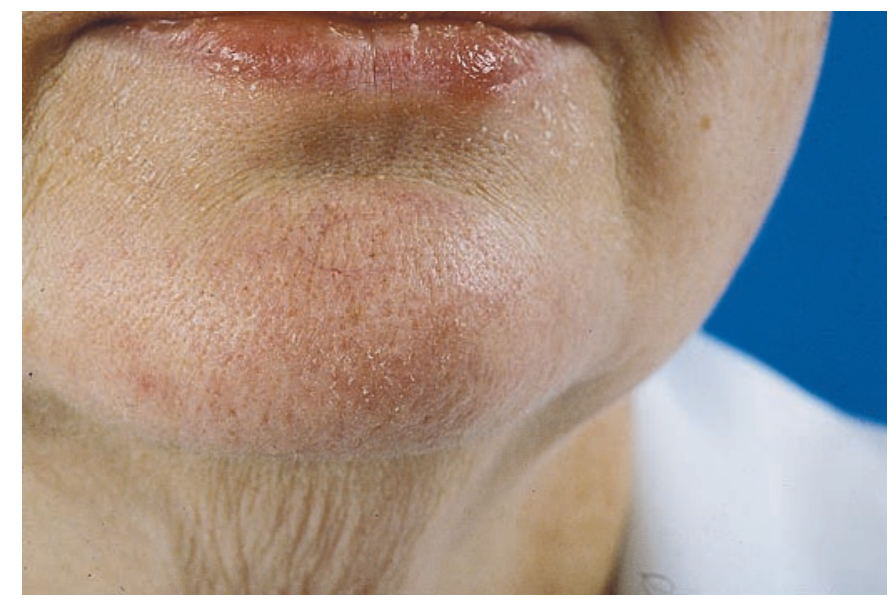

Abb. 2 Ekzematische Veränderungen durch das Mundstück im Kinnbereich.

und Missempfindungen ansah. Das Instrument wies bei näherer Inaugenscheinnahme nach Maserung, Härte und Farbton alle Merkmale einer Flöte aus Cocoboloholz auf. Die Patientin berichtete, sie habe sich gleich zwei dieser Flöten gekauft, nach kurzem aber gemerkt, dass es gar nicht nötig sei, auf die andere auszuweichen, weil man mit dem ersten Instrument „stundenlang spielen könne“. Ein Aufquellen des Holzes durch Aufnahme von Feuchtigkeit trete nicht ein, der Ton würde sich nicht verändern. Dafür würde das aus der Atemluft stammende Kondenswasser während und nach dem Spielen in Form eines Rinnsals aus der Flöte austreten.

\section{Allergologische Untersuchungen}

Nachdem der Flötenhersteller das Holz als Cocobolo bestätigt hatte, wurde auf die Entnahme von Spänen verzichtet und stattdessen gleich eine Epikutantestung mit den aus früheren Untersuchungen vorliegenden Kontaktallergenen aus Dalbergia-Arten begonnen (Tab.1). Da die beschriebenen Symptome auch den Verdacht einer Allergie vom Frühtyp nahelegten, führten wir einen Scratchtest mit frisch angefertigten feinen Spänen aus authentischem Cocoboloholz, einen Pricktest mit 15 Atopenen und 
Tab. 1 Ergebnis der Testuntersuchungen

\begin{tabular}{llllll}
\hline Allergene & Konz. & $\mathbf{2 4 h}$ & $\mathbf{7 2} \boldsymbol{h}$ & $\mathbf{9 6 h}$ & $\mathbf{1 2 0} \boldsymbol{h}$ \\
\hline (R)-4-Methoxydalbergion & $1 \%$ & $\varnothing$ & $? \mathrm{a}$ & + & ++ \\
\hline (S)-4-Methoxydalbergion & $1 \%$ & $\varnothing$ & $\varnothing$ & $\varnothing$ & $\varnothing$ \\
\hline Obtusachinon & $1 \%$ & $\varnothing$ & +++ & +++ & +++ \\
\hline (R)-3,4-Dimethoxydalbergion & $0,01 \%$ & $\varnothing$ & $\varnothing$ & $\varnothing$ & $\varnothing$ \\
(S)-4,4'-Dimethoxydalbergion & $1 \%$ & $\varnothing$ & $\varnothing$ & $\varnothing$ & $\varnothing$ \\
\hline (S)-Hydroxy-4-methoxydalbergion & $1 \%$ & $\varnothing$ & $\varnothing$ & $\varnothing$ & $\varnothing$ \\
\hline
\end{tabular}

eine CAP-Untersuchung durch. Die in der Pricktestung erhaltenen Hinweise auf allergische Frühreaktionen durch Frühblüher, Gräser und Milben bestätigten sich durch folgende CAP-Befunde: Erle (Klasse I), Liesch- und Knäuelgras (Klasse II), Hausstaubund Mehlmilbe (Klasse III), Birke (Klasse IV). Das Gesamt-IgE lag bei $62 \mathrm{KU} / \mathrm{L}$. Der Scratchtest mit den Cocobolospänen blieb bei der Patientin und drei Kontrollpersonen negativ. Im Epikutantest mit fünf Dalbergionen aus Cocobolo, verwandten Palisanderarten und einem Chinonmethid erhielten wir ausschließlich positive Testreaktionen auf die Cocobolo-Inhaltsstoffe Obtusachinon und sein korrespondierendes Chinon (R)-4-Methoxydalbergion (Tab. 1).

\section{Therapie und Verlauf}

Unter der Testung kam es zu einer Verstärkung der Lippenschwellung. Das Meiden der Flöte führte zum Abklingen der Ekzemreaktion. Beim ersatzweisen Spielen von Instrumenten aus Kirschen-, Rosen- und Ebenholz traten keine Rezidive auf.

In Absprache mit dem Flötenhersteller wurde die Anfertigung eines neuen Mundstückes aus Olivenholz, Buchenholz (mit anschließender Anpassung durch Einfärben) oder Kunststoff vereinbart. Beim Besuch des Flötenbauers im Vogtland hielt sich die Patientin für zwei Stunden in dessen Werkstatt auf. Am nächsten Tag kam es zu einem Rezidiv mit starker Schwellung der Lippen und Streuherden des Ekzems auf benachbarte Bezirke im Gesicht, obwohl kein direkter Kontakt mit dem Cocoboloholz bestanden hatte. Der Flötenbauer konnte sich jedoch nicht entschließen, ein anderes Holz für das Mundstück zu verwenden, da er den Klang erhalten wollte. Stattdessen lackierte er es mit einem wasserfesten Lack. Die Patientin verzichtete aber auf das weitere Benutzen der Cocoboloflöte, hatte sie doch beobachtet, dass das Kondenswasser der Atemluft aus dem Inneren der Flöte austritt. Sie hegte die berechtigte Befürchtung, die Allergene könnten in diesem Rinnsal enthalten sein und ein Wiederauftreten der Hautveränderungen provozieren. Sie legte das Instrument daher beiseite und spielt seither nur auf den anderen Flöten.

\section{Diskussion}

Auch wenn seine Bedeutung zurückgegangen ist, hat Cocobolo seinen Ruf als „Exklusivholz“ bis heute nicht verloren. Daran ändern auch die beobachteten allergischen Reaktionen nichts, die bereits im Jahre 1891 erstmals beschrieben wurden [3]. Vor dem 2. Weltkrieg stellte man etwa $90 \%$ aller Blockflöten aus Cocobolo her. Die dabei beobachtete Zunahme von Dermatitiden (im perioralen Bereich) und Cheilitiden führte in Musikzeitschriften, Fachblättern der Musikinstrumentenhersteller, dermatologischen Journalen und sogar Tageszeitungen zu heftigen Diskussionen. Im Jahre 1933 zog das Arbeits- und Wohlfahrtsministerium des Deutschen Reiches ein Verbot der Verarbeitung von Cocobolo in Erwägung [4], in England blieb ein solches Verbot längere Zeit in Kraft. Nach 1945 flaute das Interesse an diesem Holz zunächst ab, wurde aber im Laufe der zunehmenden Prosperität der Bundesbürger wieder geweckt $[5,6]$. Aus anderen Ländern Europas sowie Nordamerika liegen inzwischen ebenfalls Fallbeschreibungen über Sensibilisierungen durch Holzschmuck, Billardstöcke, Furniere und Einbaumöbel vor [7-10]. Bei einer dänischen Patientin kam es durch das Tragen eines Holzarmbandes zu einer EEM-ähnlichen Reaktion [11].

Botanisch steht das Cocoboloholz dem Rio-Palisander (D. nigra All.), Ostindisch-Palisander (D. latifolia Roxb.), Grenadill (D. melanoxylon Guill. \& Perr.) und anderen Dalbergia-Arten nahe, enthält aber im Gegensatz zu diesen als Hauptallergen ein Chinonmethid - das Obtusachinon -, dessen Sensibilisierungsvermögen jenes der ( $\mathrm{R})$ - und (S)-Dalbergione wesentlich übersteigt [6]. Zum schwächeren Allergen (R)-4-Methoxydalbergion (R-4-MD) steht Obtusachinon in einer direkten Beziehung. Durch Isomerisierung des R-4-MDs zum Obtusachinon und vice versa, wie sie bei der Biogenese in der Natur stattfindet, verläuft dieser Vorgang (engl.: rearrangement), enzymatisch gesteuert, auch in der Haut. Da man Obtusachinon bei der Isolierung aus dem Holz gewöhnlich in größerer Ausbeute (als R-4-MD) gewinnt und ihm gleichzeitig die höhere Sensibilisierungspotenz zukommt [6], erklärt sich auch, warum Obtusachinon regelmäßig in der Epikutantestung eine stärkere Testreaktion hervorruft als R-4-MD. So auch in unserem Fall. Kreuzreaktionen auf andere Dalbergione werden kaum beobachtet. Auch mit dem stärksten aller sensibilisierenden Dalbergione, dem (R)-3,4-Dimethoxydalbergion aus der botanisch verwandten Art Machaerium scleroxylon Tul. (Pao ferro) zeigte unsere Patientin keine Kreuzreaktion (Tab.1).

Eine Allergie vom Frühtyp gegenüber Cocobolo ließ sich bei der atopischen Patientin nicht nachweisen.

Die früher üblichen Bezeichnungen Dalbergia retusa Hemsl. und D. obtusa Lec. sind obsolet. Heute rechnet man der Spezies Cocobolo aufgrund der holzanatomischen Struktur vier Arten zu: Dalbergia granadillo Pittier, $D$. hypoleuca Pittier, $D$. lineata Pittier und D. retusa Hemsl. Da sich diese Arten jedoch in ihren Inhaltsstoffen nicht unterscheiden, differenziert man sie nicht weiter, sondern subsummiert sie unter der Bezeichung Dalbergia spc. „Mittelamerika“ [12].

Mit dem hier vorgestellten Fall bestätigt sich erneut die stark sensibilisierende Wirkung des Cocoboloholzes. Die in der 72-Stundenablesung mit ?a eingestufte Testreaktion auf R-4-MD verstärkte sich in den darauf folgenden Tagen auf ++, während die bereits in der 24-Stundenablesung +++-fach positive Reaktion auf Obtusachinon weiterhin stark zunahm und auch nach zwei Wochen noch deutlich sichtbar war. Das nach dem Werkstattbesuch auftretende, aerogen ausgelöste Rezidiv unter- 


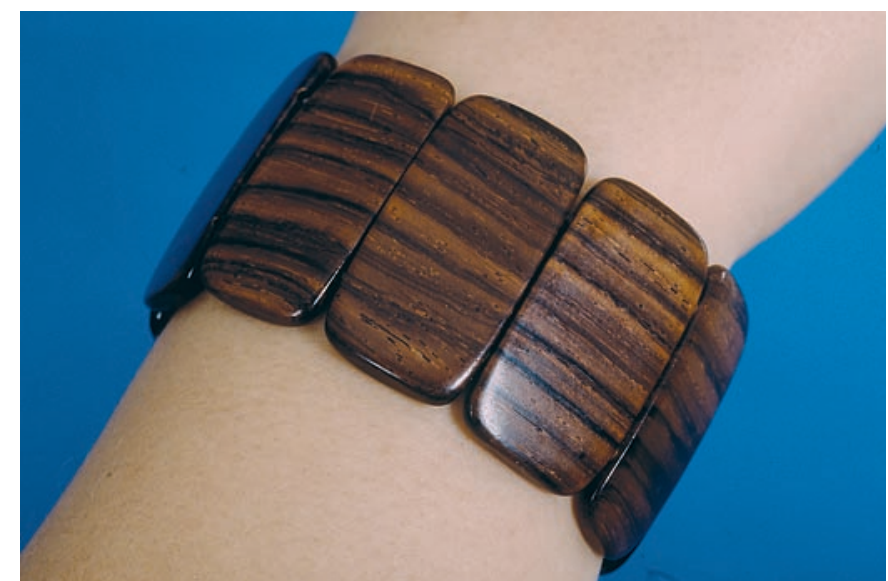

Abb. 3 Ein im Urlaub erstandenes Cocobolo-Holzarmband. Die Trägerin entwickelte ein Kontaktekzem am Unterarm.

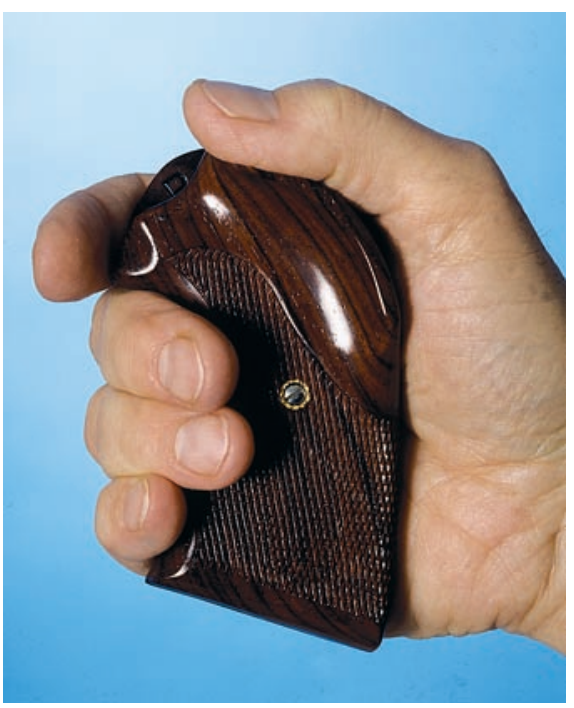

Abb. 4 Pistolengriff eines amerikanischen Sportschützen. Epikutantest mit den Inhaltsstoffen des Cocoboloholzes positiv.

streicht ebenfalls die hohe Potenz der allergenen Inhaltsstoffe. Die Sensibilisierung hatte sich innerhalb eines relativ kurzen Zeitraumes eingestellt: Die Flöte war bis zur erstmaligen Vorstellung in der Klinik (August 2002) erst ein Jahr lang benutzt worden.

In vier weiteren, bisher unveröffentlichten Fällen einer Cocobolo-Kontaktallergie wurde mit den von uns zur Verfügung gestellten Dalbergionen und Obtusachinon Testuntersuchungen vorgenommen. Im ersten Fall handelte es sich um einen kanadischen Tischler mit Handekzem. Die Hautveränderungen traten nach der Verarbeitung von Cocobolo für Einbaumöbel auf. Während seiner Tätigkeit kam es zu Streuherden im Gesicht und an den Armen. Ein übersandtes Muster wurde sowohl holzanatomisch als auch durch Nachweis seiner charakteristischen Inhaltsstoffe als Cocobolo identifiziert. Der Patient reagierte bereits nach sechs Stunden so heftig auf das Obtusachinon, dass das Testpflaster entfernt werden musste. Auch R-4-MD ergab in der 72-Stundenablesung eine starke Reaktion (priv. Mitt. Dr. J. C. Mitchell, Vancouver). Im zweiten Fall entwickelte ein Professor für Pflanzenphysiologie und nebenbei begeisterter Violinspieler eine allergische Kontaktdermatitis auf seinen Geigenkinnhalter. Holz- anatomisch erwies sich der in Polen gefertigte Kinnhalter eindeutig als Dalbergia granadillo Pittier. Die Epikutantestung führte zu zweifach-positiven Reaktionen auf (R)- und (S)-4-MD und einer einfach-positiven auf (S)-4,4'-Dimethoxydalbergion, während Obtusachinon bereits in der 48-Stundenablesung eine +++-Reaktion zeigte. Möglicherweise hatte ein früherer Geigenkinnhalter aus Rio- oder Ostindisch-Palisander bestanden, worauf die Testreaktionen der beiden anderen Dalbergione einen Hinweis lieferten. Auch auf das Geigenharz (Kolophonium) war der Pflanzenphysiologe allergisch (priv. Mitt. aus Belgien). Der dritte Fall eines Ekzems am Handgelenk ereignete sich kürzlich in der Bundesrepublik. Einzelheiten der Testuntersuchungen mit den übersandten Allergenen liegen nicht vor. Das auf einer Urlaubsreise erstandene Holzarmband (Abb.3) wurde als aus Cocobolo bestehend identifiziert, die bekannten Inhaltsstoffe nachgewiesen. Im vierten Fall gelangte das Holz über einen kalifornischen Dermatologen in unsere Hände. Sein Patient hatte sich daraus einen dekorativen Griff für seine Sportpistole (Abb.4) anfertigen lassen. In der dünnschichtchromatographischen Analyse eines Chloroformextraktes ließen sich Obtusachinon und R-4-MD nachweisen. Der Epikutantest mit den übersandten Allergenen fiel positiv aus. Trotz Nachfragens konnten keine weiteren Details in Erfahrung gebracht werden, der Fall blieb ebenfalls unveröffentlicht. Nicht unerwähnt bleiben soll eine 24-jährige Neurodermitikerin aus Linz, bei der sich ein halbes Jahr nach dem Üben auf einer neuen Cocoboloflöte (zum Vorspielen auf dem Konservatorium) Ekzemreaktionen im Gesicht entwickelten. Obwohl der Schweizer Flötenbauer eine Probe des Holzes zur Verfügung stellte und die von uns isolierten Inhaltsstoffe anschließend an die Dermatologische Abteilung des Krankenhauses in Linz gesandt wurden, konnte sich die Patientin zu jenem Zeitpunkt nicht dazu durchringen, die Epikutantestung durchführen zu lassen. Danach verloren wir sie aus den Augen.

\section{Literatur}

${ }^{1}$ Gottwald H. Handelshölzer. Ihre Benennung, Bestimmung und Beschreibung. Hamburg: F. Holzmann, 1958

2 Bultmann JD, Jurd L, Turner RD. Chemical aspects of the natural marine borer resistance of Dalbergia retusa Hemsl. Naval Res Lab Bull, 1983; $1-21$

3 Stern E. Über einige Hautkrankheiten der Musiker. Münch med Wschr $1891 ; 42: 739-740$

${ }^{4}$ Anonym. Mitteilung der Gewerbekammer Plauen vom 22. 2. 1933 (aus: Jahresbericht des Verbandes Musikindustrieller, Bd 50,1933)

${ }^{5}$ Steinbrink W. Besondere Beobachtungen an allergischen Krankheiten. Ztsch ges inn Med 1950; 50: $311-314$

${ }^{6}$ Hausen B, Münster G. Cocobolo-Holz, ein vergessenenes Ekzematogen? Derm Beruf Umwelt 1983; 31: 110-117

${ }^{7}$ Rackett SC, Zug KA. Contact dermatitis to multiple exotic woods. Am J Contact Dermatitis 1997; 8: 114-117

${ }^{8}$ Ducombs G, Felix B. Bijoux en bois tropicaux et dermite de contact. La Lettre du GERDA 1998; 15: 68 - 69

${ }^{9}$ Guanche AD, Prawer S. Generalized eczematous contact dermatitis from Cocobolo wood. Am J Contact Dermatitis 2003; 14: 90 - 92

${ }^{10}$ Correale CE, Marks JG. Contact dermatitis in a woodworker. Am J Contact Dermatitis 2001; 12: 122

${ }^{11}$ Veien VK, Hausen BM. Contact dermatitis associated with an erythema-multiforme-like eruption. Am J Contact Dermatitis 2001; 11: $235-237$

12 Richter HG, Krause UJ, Muche C. Dalbergia congestiflora Standley: wood structure and physico-chemical properties compared with other Central American species of Dalbergia. Int Assoc Wood Anatom J 1996; 17: $327-341$ 